Supporting Information for

\title{
The Behavior of Trispyrazolylborato-Metal(II)-Flavonolate Complexes as Functional Models for Bacterial Quercetinase - Assessment of the Metal Impact
}

Santina Hoof and Christian Limberg*

Institut für Chemie, Humboldt-Universität zu Berlin, Brook-Taylor-Str. 2, 12489 Berlin, Germany

*corresponding author: christian.limberg@chemie.hu-berlin.de

亩 $+4930-20937382$

$\underline{\text { Table of Contents }}$

Crystal Structure Analysis

NMR Spe ctra

Cyclic Volt ammetry

UV-vis Spectra

References

14 


\section{Crystal Structure Analysis}

The data collections were performed with a BRUKER D8 VENTURE area detector with Mo-K $\alpha$ radiation $(\lambda=0.71073 \AA$ A $)$. Multi-scan absorption corrections implemented in SADABS ${ }^{1}$ were applied to the data. The structures were solved by intrinsic phasing method (SHELXT-2013) ${ }^{2}$ and refined by full matrix least square procedures based on F2 with all measured reflections (SHELXL-2014 in the graphical user interface SHELXle $)^{3}$ with anisotropic temperature factors for all non-hydrogen atoms. Hydrogen atoms were added geometrically and refined by using a riding model except for the B- $H$ atoms which were found in the electron density map and freely refined. CCDC 1917849-1917856 contain the supplementary crystallographic data for this paper. These data can be obtained free of charge from The Cambridge Crystallographic Data Centre via www.ccdc.cam.ac.uk/data_request/cif.

\section{Crystallographic Data for [Tp $\left.{ }^{\mathrm{Mes}} \mathrm{NiBr}\right]$ (CCDC 1917854):}

$\mathrm{C}_{36} \mathrm{H}_{40} \mathrm{BBrN}_{6} \mathrm{Ni}, M_{r}=706.17$, triclinic, $P 1$, pink block, $0.40 \times 0.25 \times 0.11 \mathrm{~mm}, a=9.2282(7) \AA, b=$ 10.8784(8) $\AA, c=17.5538(11) \AA, \alpha=102.271(3)^{\circ}, \beta=98.432(3)^{\circ}, \gamma=95.447(3)^{\circ}, V=1688.8(2) \AA^{3}, Z=$ 2, $\rho=1.389 \mathrm{~g} \cdot \mathrm{cm}^{-3}, \mu=1.792 \mathrm{~mm}^{-1}, T=100(2) \mathrm{K}, \Theta=2.37-26.43^{\circ}, F_{000}=732$, reflections collected 41372, reflections unique $13618\left[R_{\text {int }}=0.0356\right]$, GooF $=0.980, R_{1}=0.0238, w R_{2}=0.0548$, largest diff. peak and hole $0.263 /-0.468 \mathrm{e}^{-3}$.

This compound is isostructural to the related $\mathrm{Tp}^{\mathrm{Mes}} \mathrm{NiCl}$ complex (CCDC 226463). ${ }^{4}$

Crystallographic Data for [Tp ${ }^{\text {Mes }}$ MnFla] (CCDC 1917856):

$\mathrm{C}_{51} \mathrm{H}_{49} \mathrm{BMnN}_{6} \mathrm{O}_{3}, M_{r}=859.71$, triclinic, $P$-1, olive fragment, $0.32 \times 0.17 \times 0.06 \mathrm{~mm}, a=12.0949(14) \AA$, $b=12.1035(15) \AA, c=18.281(2) \AA, \alpha=71.342(7)^{\circ}, \beta=86.708(7)^{\circ}, \gamma=61.254(6)^{\circ}, V=2208.0(5) \AA^{3}, Z=$ 2, $\rho=1.293 \mathrm{~g} \cdot \mathrm{cm}^{-3}, \mu=0.350 \mathrm{~mm}^{-1}, T=100(2) \mathrm{K}, \Theta=2.37-25.10^{\circ}, F_{000}=902$, reflections collected 15892 , reflections unique $7200\left[R_{\text {int }}=0.0784\right]$, GooF $=1.026, R_{1}=0.0738, w R_{2}=0.1821$, largest diff. peak and hole $0.974 /-0.694 \mathrm{e}^{-3}$.

Crystallographic Data for [Tp ${ }^{\text {Mes }}$ FeFla] (CCDC 1917850):

$\mathrm{C}_{51} \mathrm{H}_{49} \mathrm{BFeN}_{6} \mathrm{O}_{3}, M_{r}=860.62$, triclinic, $P-1$, brown plate, $0.19 \times 0.11 \times 0.08 \mathrm{~mm}, a=12.0492(10) \AA, b=$ $12.0631(10) \AA, c=18.2882(14) \AA, \alpha=87.195(3)^{\circ}, \beta=71.572(3)^{\circ}, \gamma=61.533(3)^{\circ}, V=2200.4(3) \AA^{3}, Z=$ 2, $\rho=1.299 \mathrm{~g} \cdot \mathrm{cm}^{-3}, \mu=0.393 \mathrm{~mm}^{-1}, T=100(2) \mathrm{K}, \Theta=2.37-25.41^{\circ}, F_{000}=904$, reflections collected 68927, reflections unique $8082\left[R_{\text {int }}=0.0505\right]$, GooF $=1.088, R_{1}=0.0572, w R_{2}=0.1271$, largest diff. peak and hole $1.892 /-0.533 \mathrm{e}^{-3}$.

Crystallographic Data for [Tp ${ }^{\text {Mes }}$ CoFla] (CCDC 1917849):

$\mathrm{C}_{51} \mathrm{H}_{49} \mathrm{BCoN}_{6} \mathrm{O}_{3}, M_{r}=863.70$, triclinic, $P$-1, yellow plate, $0.18 \times 0.17 \times 0.06 \mathrm{~mm}, a=11.9647(13) \AA, b=$ 12.0059(12) $\AA, c=18.2765(17) \AA, \alpha=71.511(4)^{\circ}, \beta=87.391(4)^{\circ}, \gamma=61.898(4)^{\circ}, V=2179.3(4) \AA^{3}, Z=$ 2, $\rho=1.316 \mathrm{~g} \cdot \mathrm{cm}^{-3}, \mu=0.445 \mathrm{~mm}^{-1}, T=100(2) \mathrm{K}, \Theta=2.37-25.39^{\circ}, F_{000}=906$, reflections collected 33905 , reflections unique $7943\left[R_{\text {int }}=0.0681\right]$, GooF $=1.064, R_{1}=0.0784, w R_{2}=0.2207$, largest diff. peak and hole $1.760 /-0.510$ e $\AA^{-3}$.

Crystallographic Data for [Tp ${ }^{\text {Mes }}$ NiFla] (CCDC 1917851): 
$\mathrm{C}_{51} \mathrm{H}_{49} \mathrm{BN}_{6} \mathrm{NiO}_{3}, M_{r}=863.48$, triclinic, $P-1$, green plate, $0.18 \times 0.08 \times 0.06 \mathrm{~mm}, a=11.8528(8) \AA, b=$ $11.9752(8) \AA, c=18.2600(13) \AA, \alpha=71.748(3)^{\circ}, \beta=87.713(3)^{\circ}, \gamma=62.145(3)^{\circ}, V=2158.7(3) \AA^{3}, Z=2$, $\rho=1.328 \mathrm{~g} \cdot \mathrm{cm}^{-3}, \mu=0.501 \mathrm{~mm}^{-1}, T=100(2) \mathrm{K}, \Theta=2.37-25.76^{\circ}, F_{000}=908$, reflections collected 44502, reflections unique $8235\left[R_{\text {int }}=0.0855\right]$, GooF $=1.032, R_{1}=0.0521, w R_{2}=0.1204$, largest diff. peak and hole $1.064 /-0.591 \mathrm{e}^{-3}$.

\section{Crystallographic Data for [Tp ${ }^{\text {Mes }}$ ZnFla] (CCDC 1917853):}

$\mathrm{C}_{51} \mathrm{H}_{49} \mathrm{BN}_{6} \mathrm{O}_{3} \mathrm{Zn}, M_{r}=870.14$, triclinic, $P-1$, yellow plate, $0.13 \times 0.08 \times 0.04 \mathrm{~mm}, a=11.9576(7) \AA, b=$ 12.0195(7) $\AA, c=18.2637(10) \AA, \alpha=72.060(3)^{\circ}, \beta=87.393(3)^{\circ}, \gamma=61.706(2)^{\circ}, V=2183.1(2) \AA^{3}, Z=2$, $\rho=1.324 \mathrm{~g} \cdot \mathrm{cm}^{-3}, \mu=0.614 \mathrm{~mm}^{-1}, T=100(2) \mathrm{K}, \Theta=2.36-25.39^{\circ}, F_{000}=912$, reflections collected 61015 , reflections unique $8028\left[R_{\text {int }}=0.0736\right]$, GooF $=1.057, R_{1}=0.0465, w R_{2}=0.1066$, largest diff. peak and hole $1.202 /-0.419 \mathrm{e}^{-3}$.

\section{Crystallographic Data for [Tp $\left.{ }^{\text {Mes }} \mathrm{NiDep}\right] \cdot \mathrm{CH}_{3} \mathrm{CN}$ (CCDC 1917855):}

$\mathrm{C}_{50} \mathrm{H}_{49} \mathrm{BN}_{6} \mathrm{NiO}_{4} \cdot \mathrm{CH}_{3} \mathrm{CN}, M_{r}=908.52$, monoclinic, $P 2_{1} / \mathrm{n}$, blue block, $0.20 \times 0.09 \times 0.08 \mathrm{~mm}, a=$ 11.325(9) $\AA, b=19.757(14) \AA, c=20.873(17) \AA, \beta=92.433(19)^{\circ}, V=4666(6) \AA^{3}, Z=4, \rho=1.293 \mathrm{~g} \cdot \mathrm{cm}^{-}$ ', $\mu=0.469 \mathrm{~mm}^{-1}, T=100(2) \mathrm{K}, \Theta=2.21-25.39^{\circ}, F_{000}=1912$, reflections collected 66667 , reflections unique $8575\left[R_{\text {int }}=0.1417\right], \mathrm{GooF}=1.022, R_{1}=0.0465, w R_{2}=0.0934$, largest diff. peak and hole 0.320 $/-0.443$ e $\AA^{-3}$.

\section{Crystallographic Data for [Tp $\left.{ }^{\text {Mes }} \mathrm{NiSal}\right] \cdot 2 \mathrm{CH}_{3} \mathrm{CN}$ (CCDC 1917852):}

$\mathrm{C}_{43} \mathrm{H}_{44} \mathrm{BN}_{6} \mathrm{NiO}_{3} \cdot 2 \mathrm{CH}_{3} \mathrm{CN}, M_{\mathrm{r}}=844.47$, triclinic, $P$-1, blue block, $0.34 \times 0.24 \times 0.10 \mathrm{~mm}, a=11.2562(10)$ $\AA, b=12.4028(13) \AA, c=17.0050(17) \AA, \alpha=100.340(4)^{\circ}, \beta=100.629(4)^{\circ}, \gamma=94.287(4)^{\circ}, V=2281.1(4)$ $\AA^{3}, Z=2, \rho=1.229 \mathrm{~g} \cdot \mathrm{cm}^{-3}, \mu=0.474 \mathrm{~mm}^{-1}, T=100(2) \mathrm{K}, \Theta=2.36-25.77^{\circ}, F_{000}=890$, reflections collected 72891, reflections unique $8711\left[R_{\text {int }}=0.0319\right]$, GooF $=1.035, R_{1}=0.0563, w R_{2}=0.1436$, largest diff. peak and hole $2.014 /-0.990 \mathrm{e}^{-3}$.

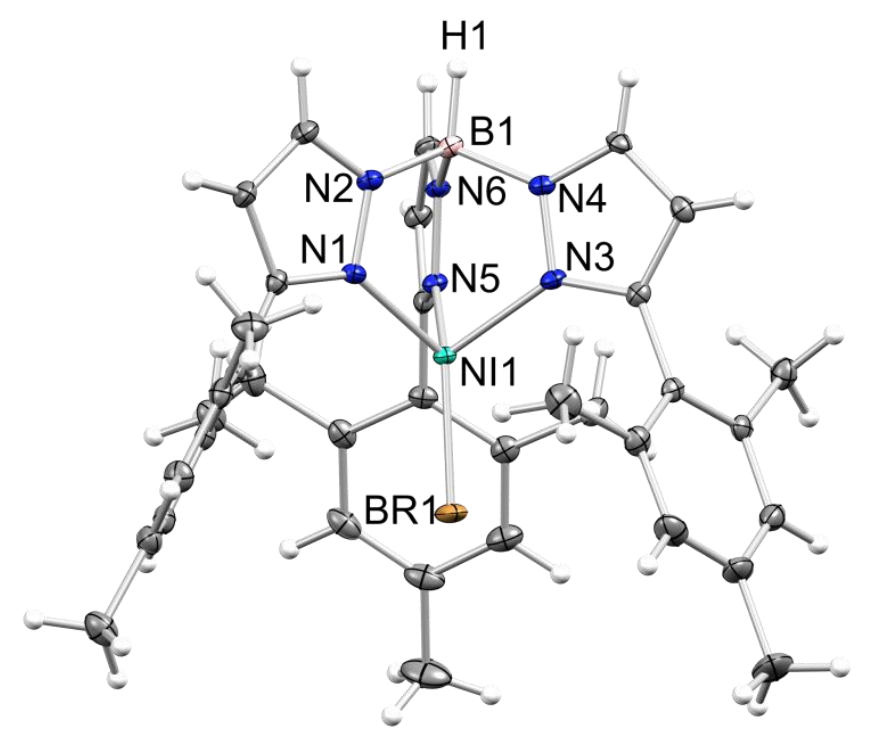

Figure S1: Molecular structure of $\mathrm{Tp}^{\mathrm{Mes}} \mathrm{NiBr}$ as determined by single crystal X-ray diffraction. Thermal ellipsoids are shown at the $50 \%$ probability level; selected bond lengths $[\AA ̊]$ and angles $\left[^{\circ}\right]: \operatorname{Br}(1)-\mathrm{Ni}(1)$ 2.2784(5), Ni(1)-N(5) 1.985(2), Ni(1)-N(1) 1.989(2), Ni(1)-N(3) 1.995(2), B(1)-H(1) 1.13(4), N(5)-Ni(1)-N(1) 
91.57(10), N(5)-Ni(1)-N(3) 90.46(10), N(1)-Ni(1)-N(3) 93.12(10), N(5)-Ni(1)-Br(1) 127.23(7), N(1)-Ni(1)-Br(1) 125.20(7), N(3)-Ni(1)-Br(1) 119.54(7).

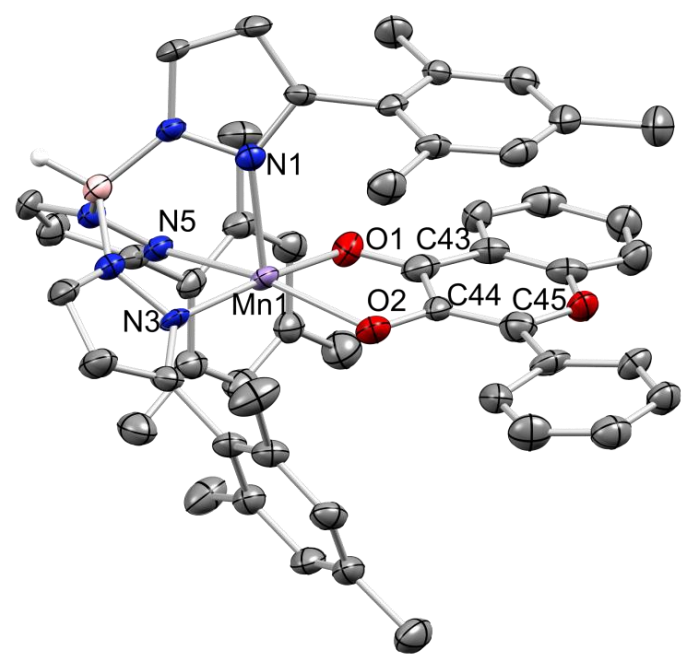

Figure S2: Molecular structure of $\mathrm{Tp}^{\mathrm{Mes}} \mathrm{MnFla}(\mathbf{1})$ as determined by single crystal X-ray diffraction. Thermal ellipsoids are shown at the $50 \%$ probability level; hydrogen atoms (except $\mathrm{B}-\mathrm{H} 1$ ) are omitted for clarity.

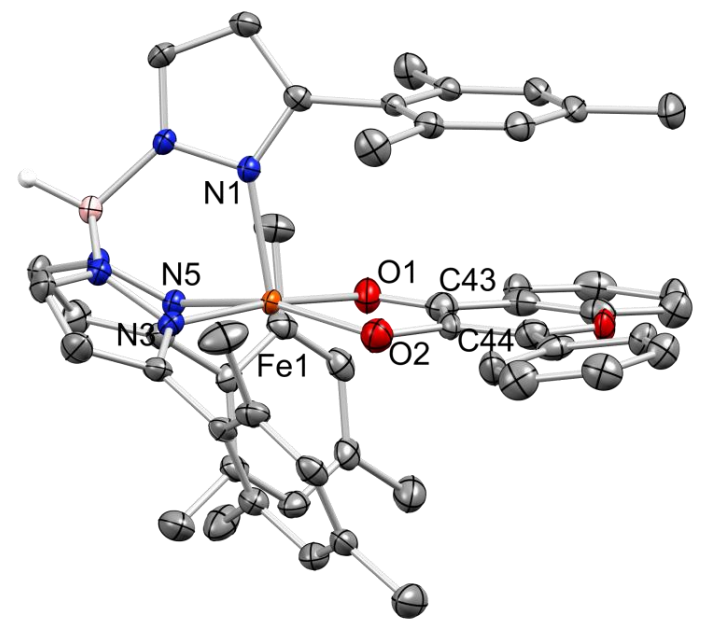

Figure S3: Molecular structure of $\mathrm{Tp}^{\text {Mes }} \mathrm{FeFla} \mathrm{(2)} \mathrm{as} \mathrm{determined} \mathrm{by} \mathrm{single} \mathrm{crystal} \mathrm{X-ray} \mathrm{diffraction.} \mathrm{Thermal}$ ellipsoids are shown at the $50 \%$ probability level; hydrogen atoms (except $\mathrm{B}-\mathrm{H} 1$ ) are omitted for clarity. 


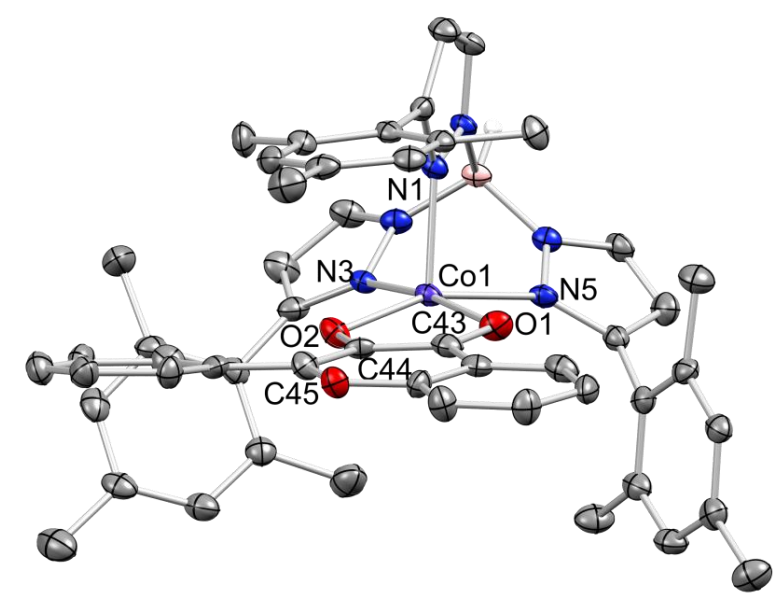

Figure S4: Molecular structure of $\mathrm{Tp}^{\mathrm{Mes}} \mathrm{CoFla}$ (3) as determined by single crystal X-ray diffraction. Thermal ellipsoids are shown at the $50 \%$ probability level; hydrogen atoms (except B-H1) are omitted for clarity.

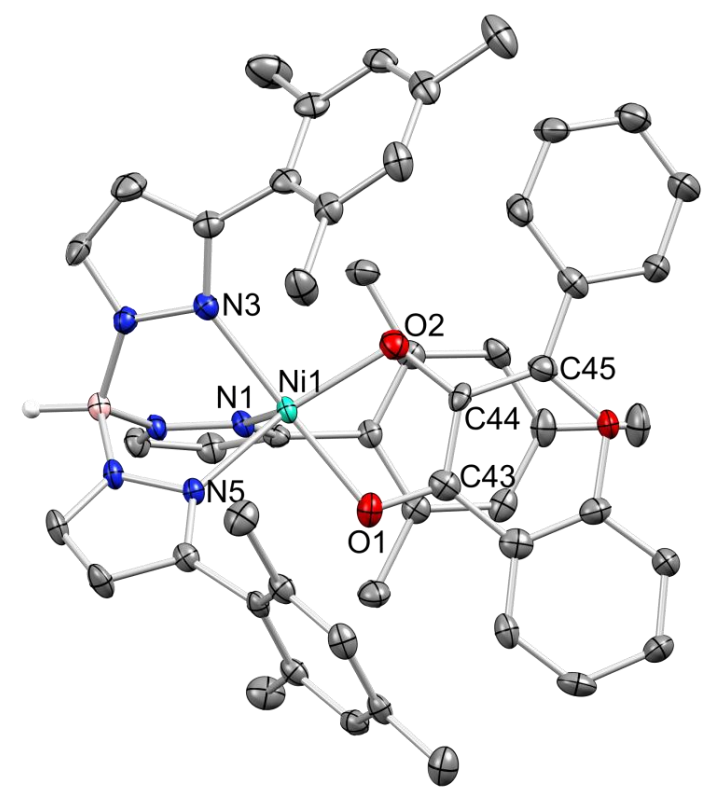

Figure S5: Molecular structure of $\mathrm{Tp}^{\mathrm{Mes}} \mathrm{NiFla}$ (4) as determined by single crystal X-ray diffraction. Thermal ellipsoids are shown at the $50 \%$ probability level; hydrogen atoms (except B-H1) are omitted for clarity. 


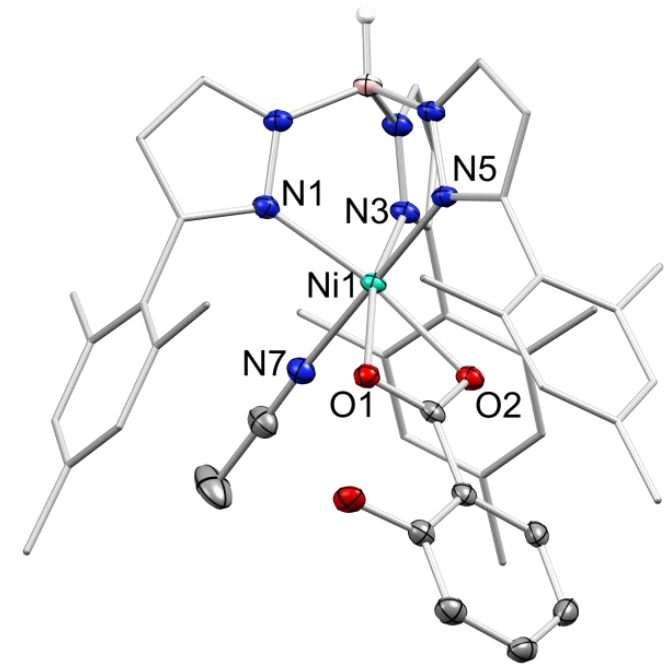

Figure S6: Molecular structure of $\mathrm{Tp}^{\mathrm{Mes}} \mathrm{NiSal}(\mathbf{7})$ as determined by single crystal X-ray diffraction. Thermal ellipsoids are shown at the 50\% probability level; hydrogen atoms (except $\mathrm{B}-\mathrm{H} 1$ ) and one acetonitrile solvent molecule are omitted for clarity; selected bond lengths $[\AA \AA]$ and angles [ $\left.{ }^{\circ}\right]$ : $\mathrm{Ni}(1)-\mathrm{O}(1) 2.1181(18)$, $\mathrm{Ni}(1)-\mathrm{O}(2)$ 2.1114(19), Ni(1)-C(37) 2.447(3), Ni(1)-N(1) 2.071(2), Ni(1)-N(3) 2.068(2), Ni(1)-N(5) 2.120(2), $\mathrm{Ni}(1)-\mathrm{N}(7)$ 2.101(2), O(1)-C(37) 1.269(3), O(2)-C(37) 1.273(3), O(2)-Ni(1)-O(1) 62.53(7), N(1)-Ni(1)-O(1) 104.23(8), N(3)-Ni(1)-O(1) 165.36(8), O(1)-Ni(1)-N(5) 93.00(8), N(7)-Ni(1)-O(1) 85.59(8), N(1)-Ni(1)-O(2) 166.68(8), N(3)-Ni(1)-O(2) 102.91(8), O(2)-Ni(1)-N(5) 93.47(8), N(7)-Ni(1)-O(2) 86.36(8), N(3)-Ni(1)-N(1) 90.27(9), N(3)-Ni(1)-N(7) 92.20(9), N(1)-Ni(1)-N(7) 91.28(9), N(3)-Ni(1)-N(5) 89.31(8), N(1)-Ni(1)-N(5) 88.54(9), N(7)-Ni(1)-N(5) 178.49(8). 
NMR Spectra
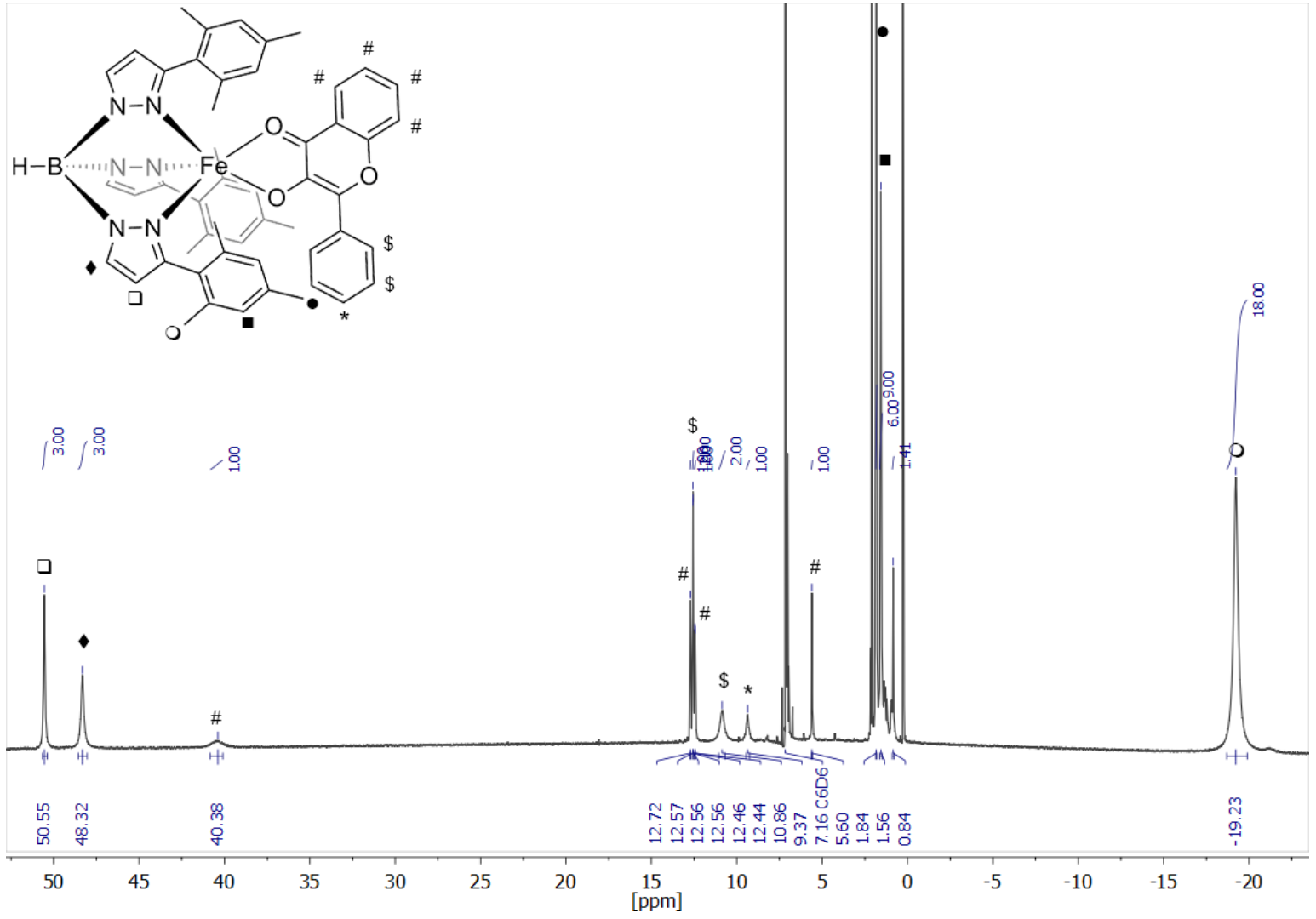

Figure S7: ${ }^{1} \mathrm{H}$ NMR (400 MHz, $\left.\mathrm{C}_{6} \mathrm{D}_{6}\right)$ spectrum of $\mathrm{Tp}^{\mathrm{Mes}} \mathrm{FeFla}$ (2).

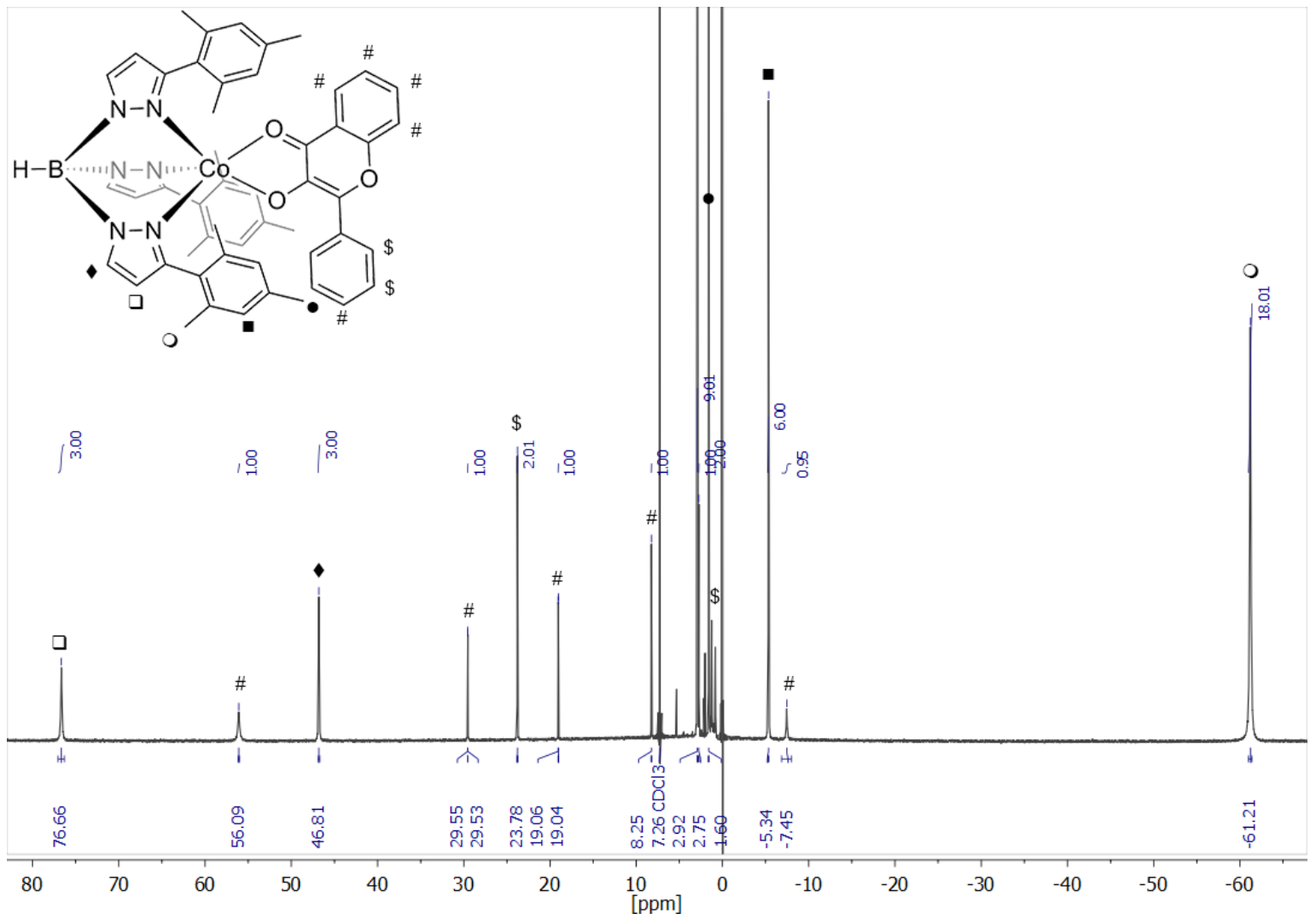

Figure S8: ${ }^{1} \mathrm{H} N M R\left(500 \mathrm{MHz}, \mathrm{CDCl}_{3}\right)$ spectrum of $\mathrm{Tp}^{\mathrm{Mes}} \mathrm{CoFla} \mathrm{(3).}$ 

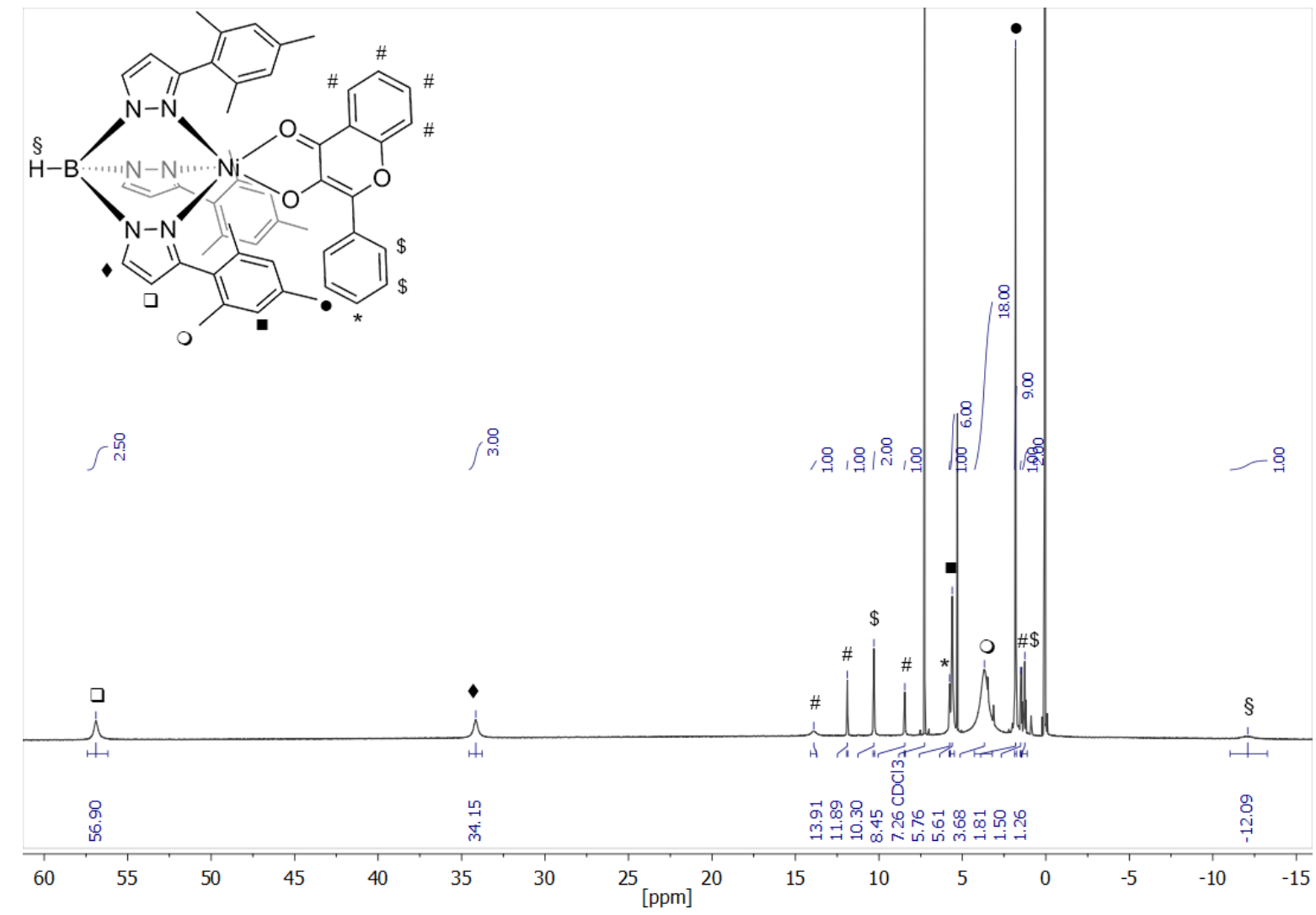

Figure S9: ${ }^{1} \mathrm{HNMR}\left(400 \mathrm{MHz}, \mathrm{CDCl}_{3}\right.$ ) spectrum of $\mathrm{Tp}^{\text {Mes }} \mathrm{NiFla} \mathrm{(4).}$

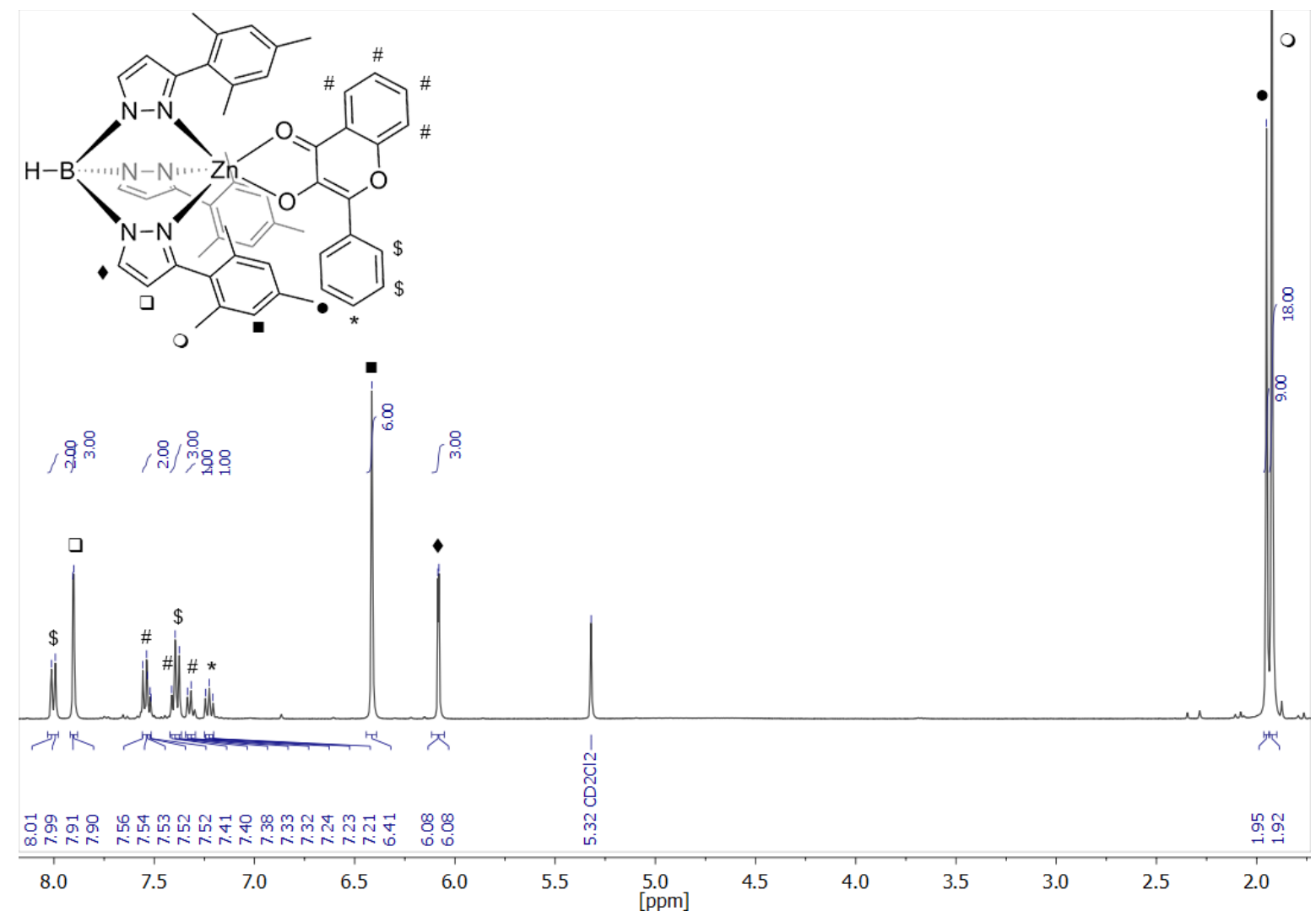

Figure S10: ${ }^{1} \mathrm{H}$ NMR (400 MHz, $\mathrm{CD}_{2} \mathrm{Cl}_{2}$ ) spectrum of $\mathrm{Tp}^{\mathrm{Mes}} \mathrm{ZnFla} \mathrm{(5).}$ 

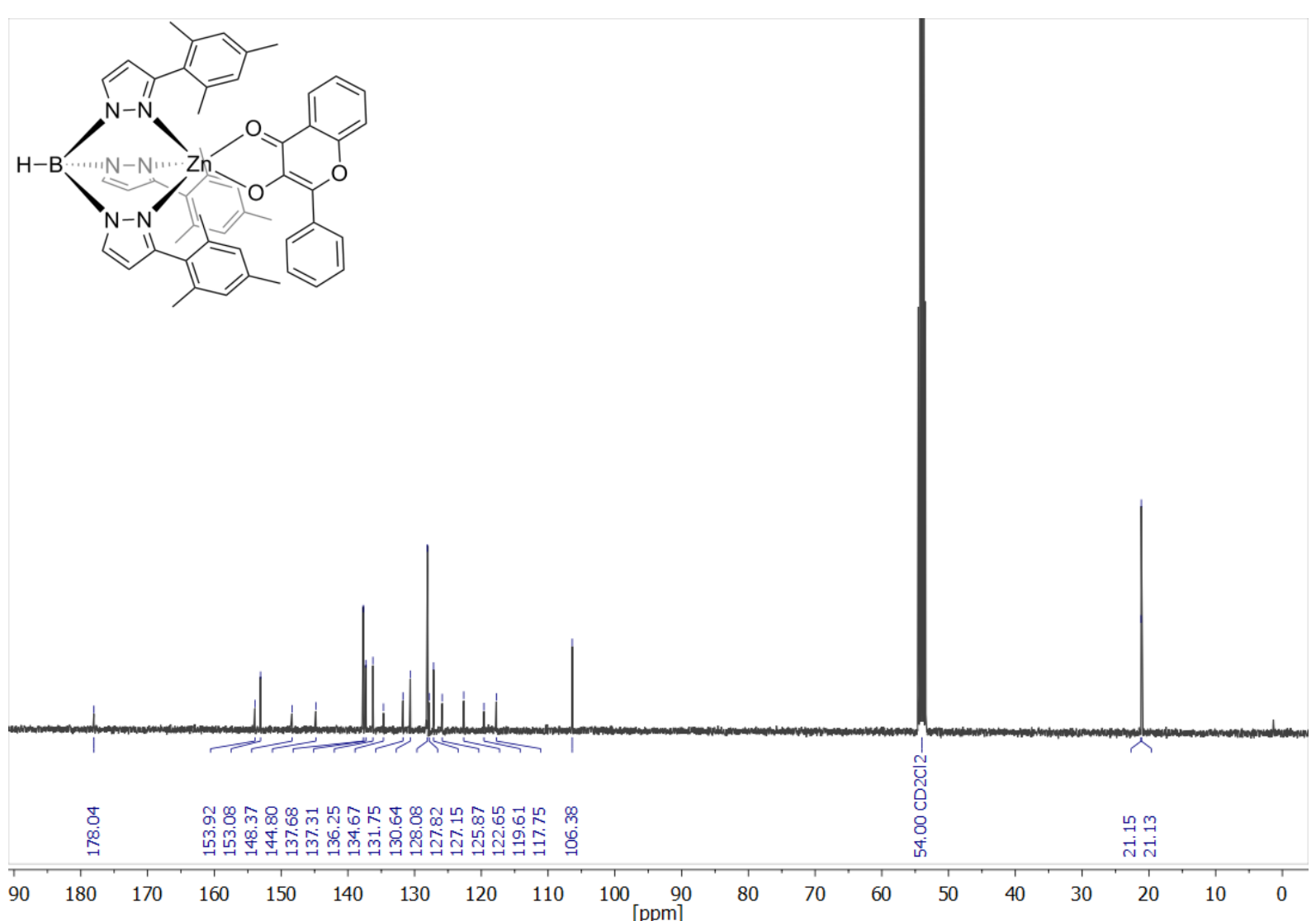

Figure S11: ${ }^{13} \mathrm{CNMR}\left(101 \mathrm{MHz}, \mathrm{CD}_{2} \mathrm{Cl}_{2}\right.$ ) spectrum of $\mathrm{Tp}^{\mathrm{Mes}} \mathrm{ZnFla} \mathrm{(5).}$
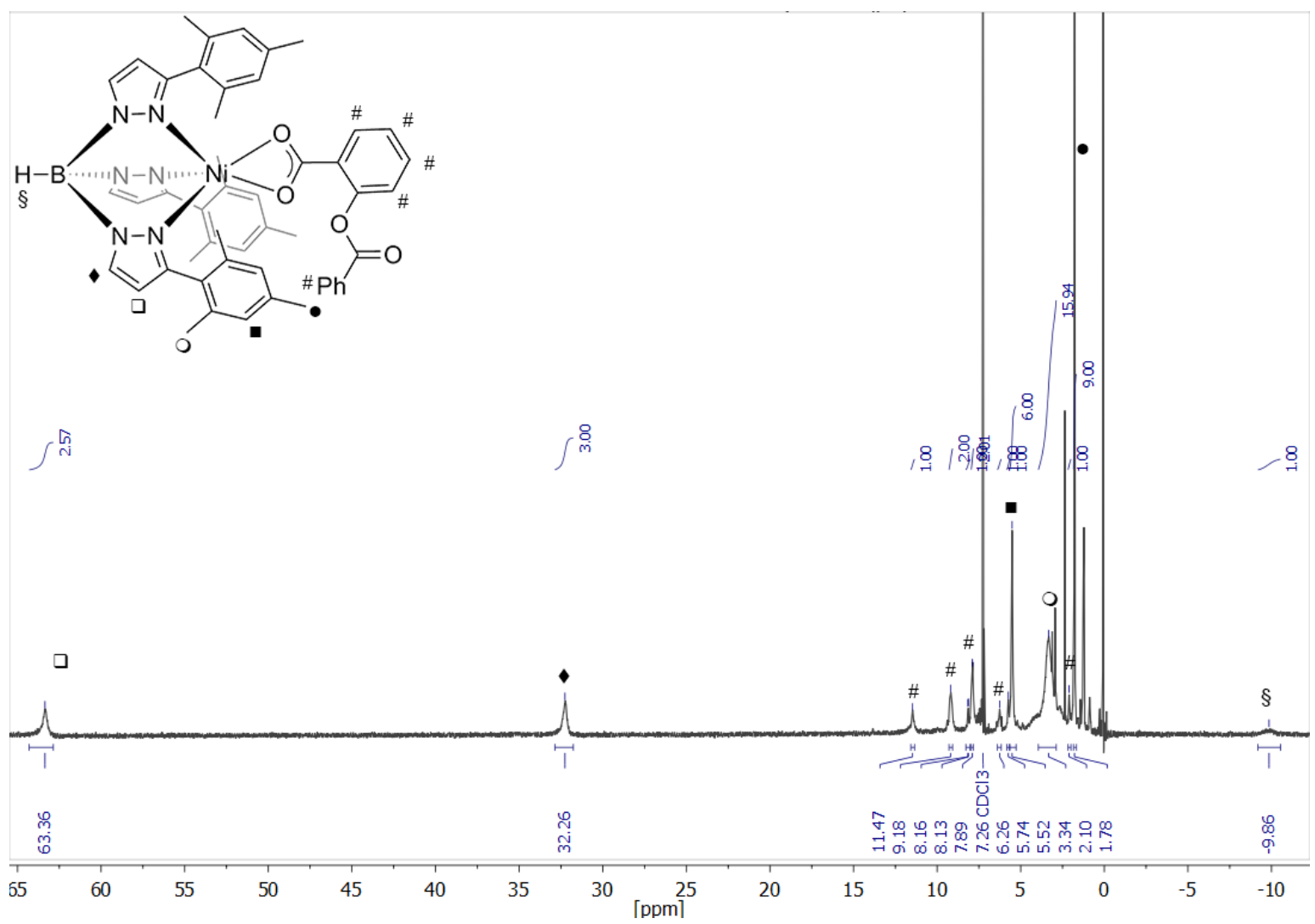

Figure S12: ${ }^{1} \mathrm{H} N M R\left(400 \mathrm{MHz}, \mathrm{CDCl}_{3}\right)$ spectrum of $\mathrm{Tp}^{\text {Mes }} \mathrm{NiDep}$ (6). 


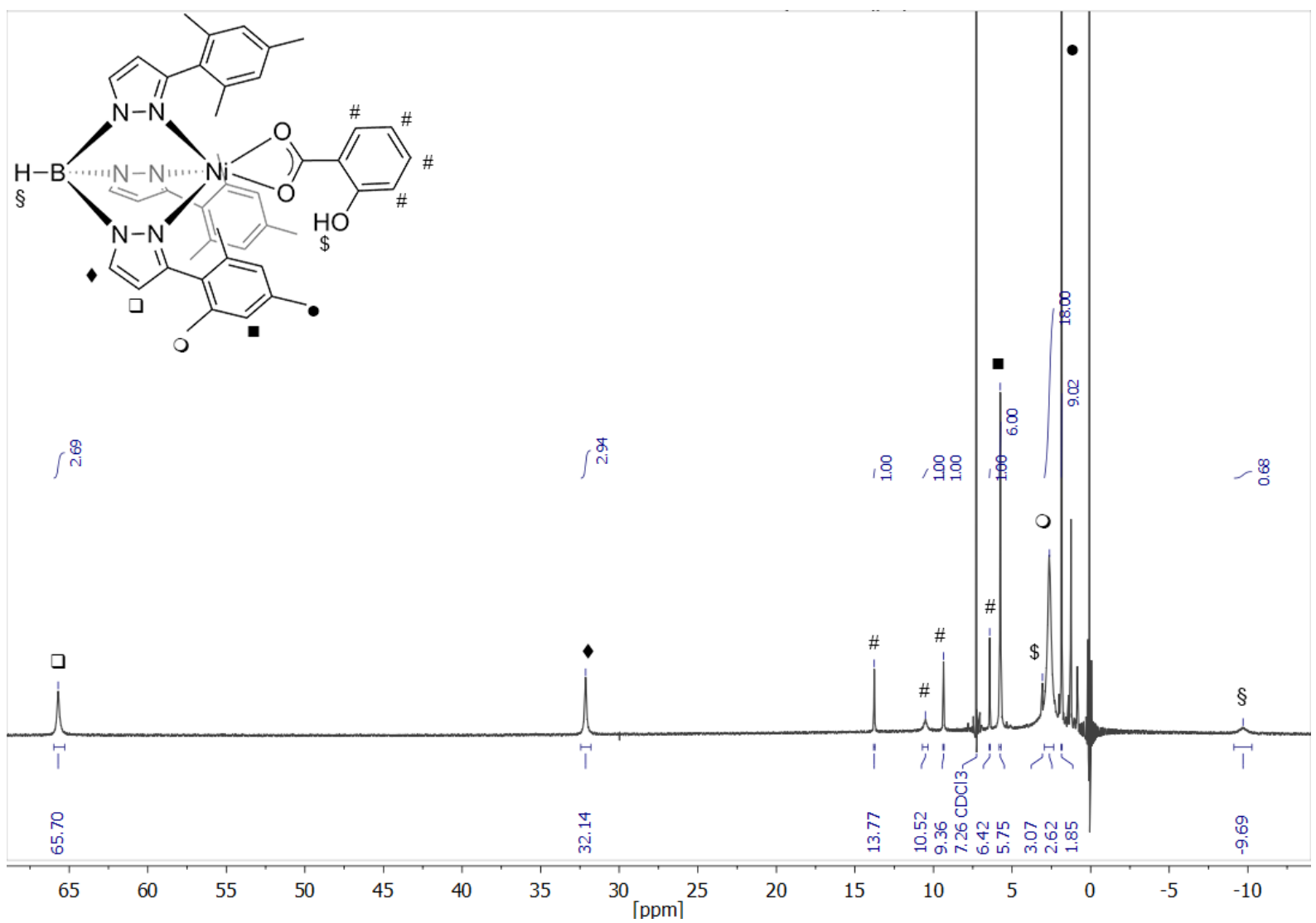

Figure S13: ${ }^{1} \mathrm{H} \mathrm{NMR}\left(400 \mathrm{MHz}, \mathrm{CDCl}_{3}\right)$ spectrum of $\mathrm{Tp}^{\text {Mes }} \mathrm{NiSal}(\mathbf{7})$.

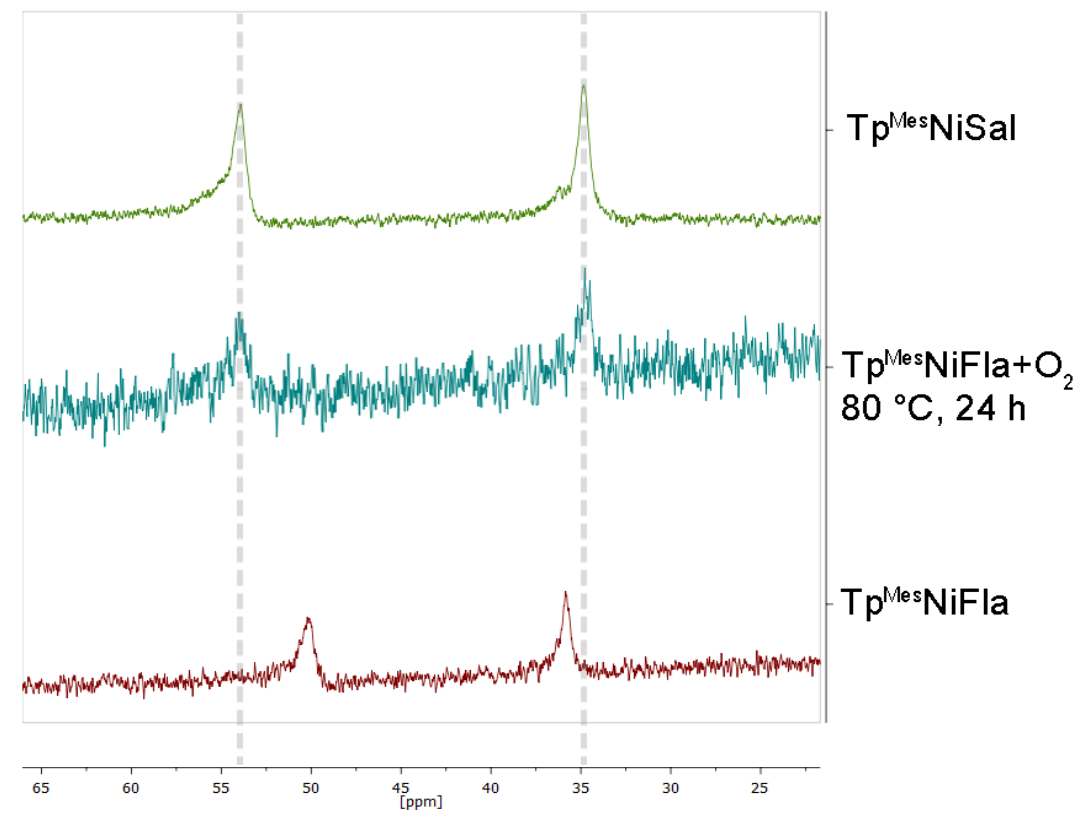

Figure S14: Section of the ${ }^{1} \mathrm{H}$ NMR $\left(300 \mathrm{MHz}, \mathrm{DMF}-\mathrm{d}_{7}\right)$ spectra, showing the signals derived from the pyrazolyl protons in 4- and 5-positions of $\mathrm{Tp}^{\mathrm{Mes}} \mathrm{NiFla} \mathrm{(4)} \mathrm{(bottom),} \mathrm{and} \mathrm{of} \mathrm{the} \mathrm{product} \mathrm{formed} \mathrm{in} \mathrm{the}$ reaction between $\mathrm{Tp}^{\mathrm{Mes}} \mathrm{NiFla}(4)$ and $\mathrm{O}_{2}$ at $80^{\circ} \mathrm{C}$ after $24 \mathrm{~h}$ (middle) in comparison with the signals observed for $\mathrm{Tp}^{\mathrm{Mes}} \mathrm{NiSal}$ (7) (top). 


\section{Cyclic Voltammetry}

a)

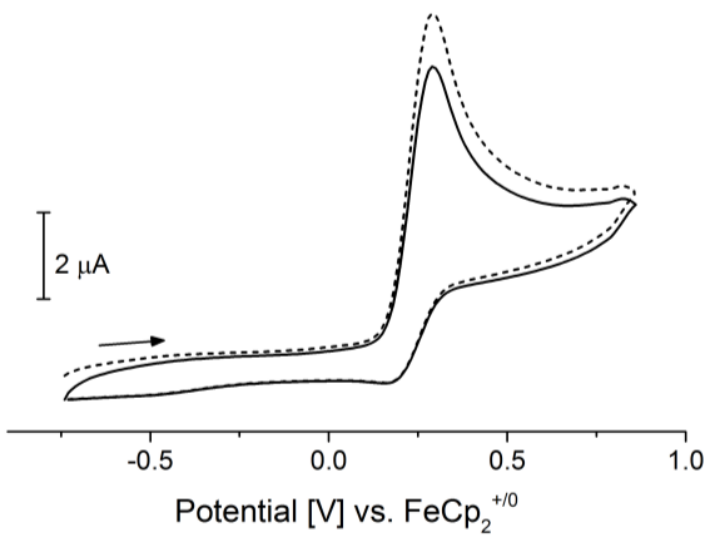

c)

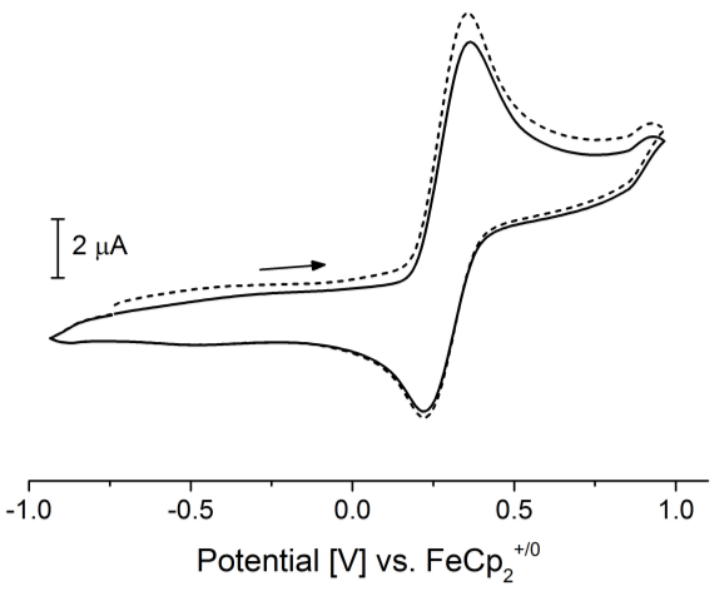

e)

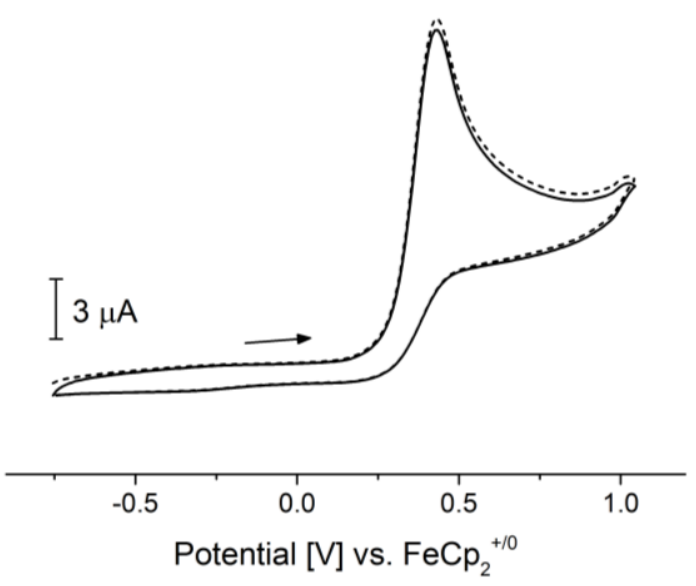

) b)

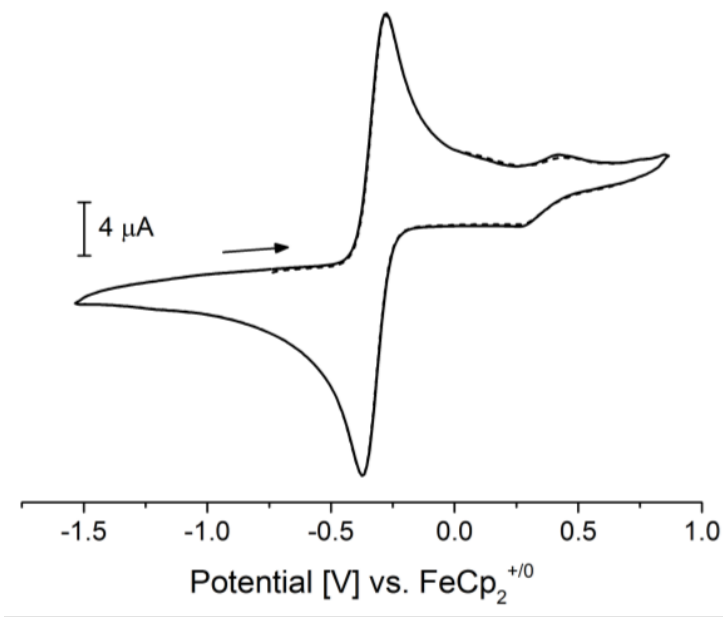

d)

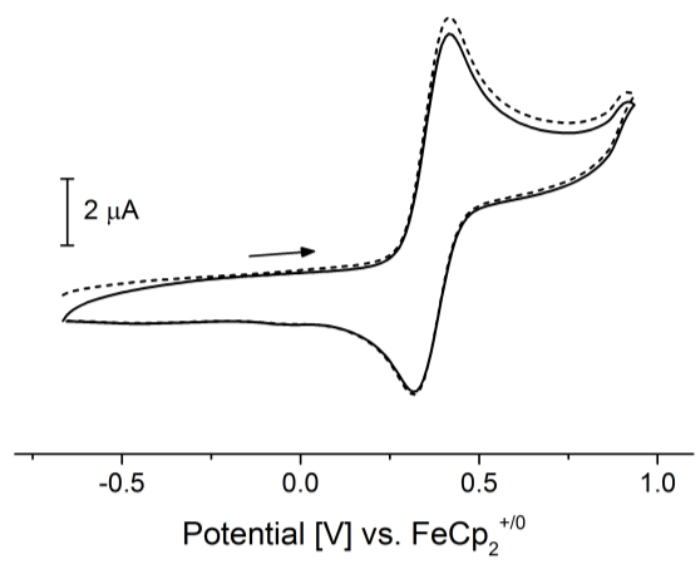

Figure S15: Cyclic voltammograms measured at a scan rate of $100 \mathrm{mV} / \mathrm{s}$ in DMF (100 mM TBAPF $61 \mathrm{mM}$ complex, … 1st scan, - 2nd scan) of a) $\mathrm{Tp}^{\mathrm{Mes}} \mathrm{MnFla}(\mathbf{1})$, b) $\mathrm{Tp}^{\mathrm{Mes}} \mathrm{FeFla}(\mathbf{2})$, c) $\mathrm{Tp}^{\mathrm{Mes}} \mathrm{CoFla}(\mathbf{3})$, d) $\mathrm{Tp}^{\mathrm{Mes}} \mathrm{NiFla}$ (4), e) $\mathrm{Tp}^{\mathrm{Mes}} \mathrm{ZnFla}(\mathbf{5})$. In case of $\mathbf{2}$ it is noted that the intensity ration between the waves belonging to the $\mathrm{Fe} / \mathrm{Fe}{ }^{\mathrm{III}}$ and $\mathrm{Fla} / \mathrm{Fla}^{+}$couples is higher than the expected 1:1. This can have several reasons, which were not investigated more closely. As the ratio decreases slightly with increasing scan rate, one contribution may be diffusion after the first redox event. 


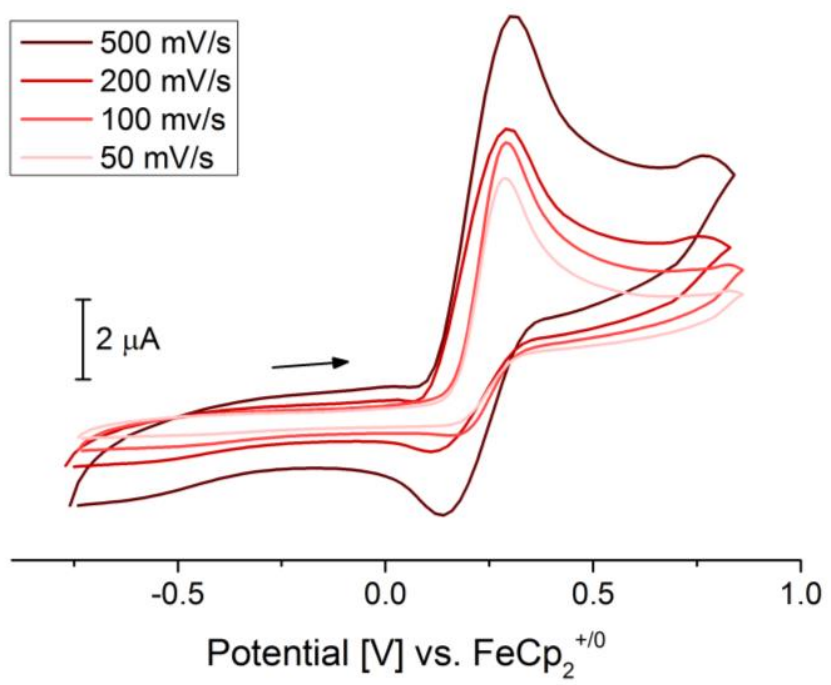

Figure S16: Cyclic voltammograms of $\mathrm{Tp}^{\mathrm{Mes}} \mathrm{MnFla}$ (1) with variable scan rates between 50 and $500 \mathrm{mV} / \mathrm{s}$ in DMF (100 mM TBAPF 6,1 mM complex).

a)

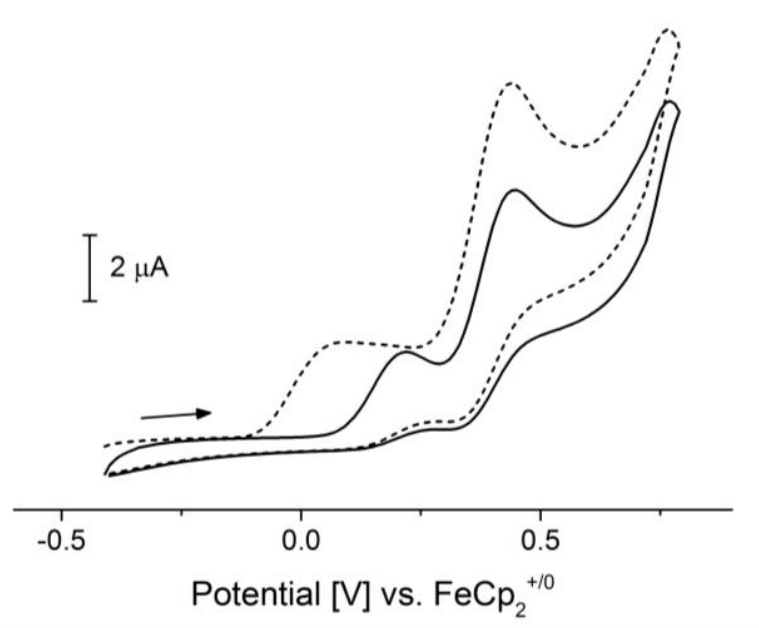

b)

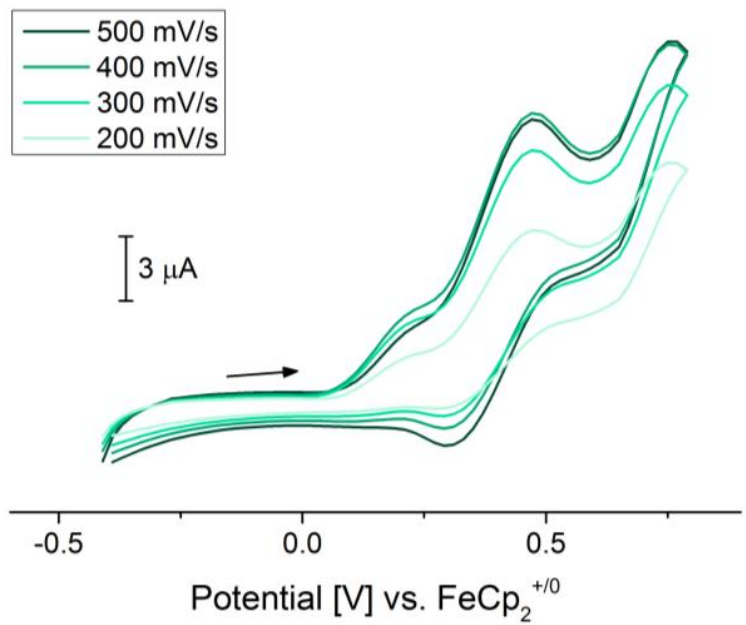

Figure S17: Cyclic voltammograms of $\mathrm{Tp}^{\mathrm{Mes}} \mathrm{NiFla}$ (4) (100 $\mathrm{mM}^{\mathrm{TBAPF}}{ }_{6}, 1 \mathrm{mM}$ complex) measured under dioxygen atmosphere a) measured at a scan rate of $100 \mathrm{mV} / \mathrm{s}$ in DMF (.... 1st scan, -2 2nd scan) and b) with variable scan rates between 200 and $500 \mathrm{mV} / \mathrm{s}$ in DMF. 
a)

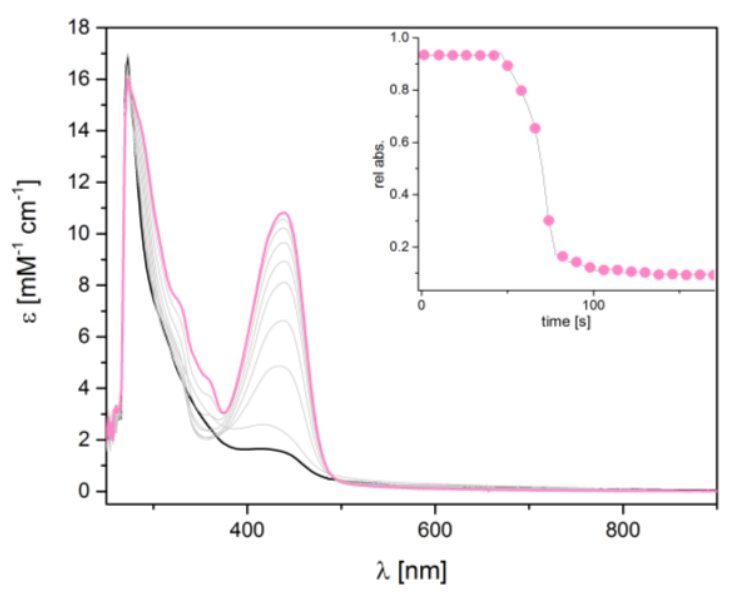

c)

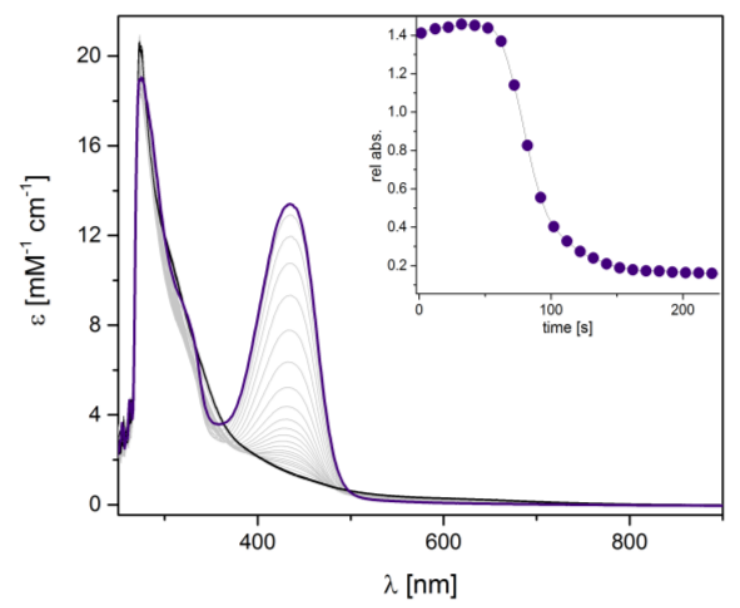

e)

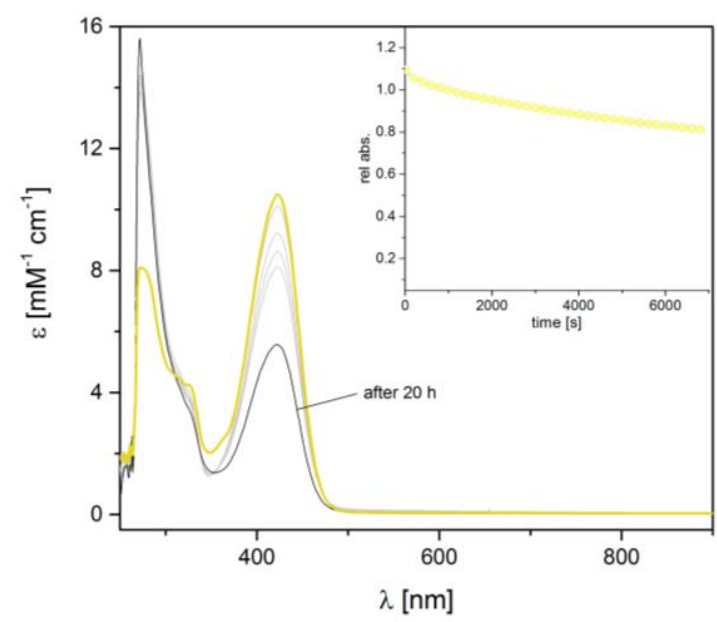

b)

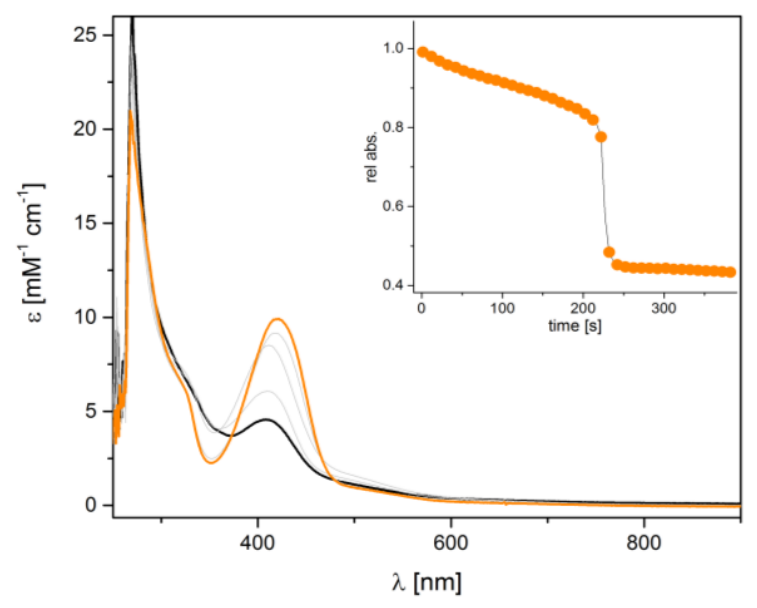

d)

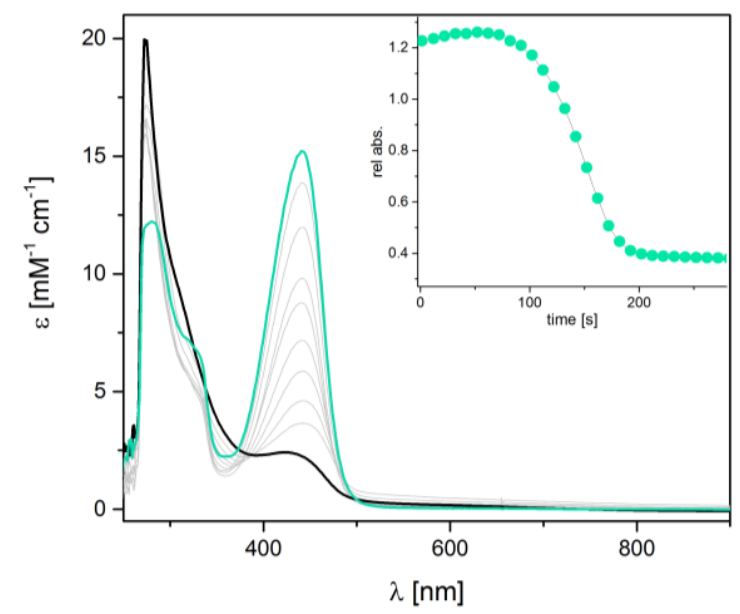

Figure S18: UV-vis spectral changes observed for the complexes $\mathrm{Tp}^{\mathrm{Mes}} \mathrm{MFla}(0.1 \mathrm{mM})$ upon addition of $\mathrm{O}_{2}$ to a DMF solution at $80^{\circ} \mathrm{C}$. Insets: Time dependent decrease of $\lambda_{\max }$ a) $\left.\mathrm{Tp}^{\mathrm{Mes}} \mathrm{MnFla}(\mathbf{1}), \mathrm{b}\right) \mathrm{Tp}^{\mathrm{Mes}} \mathrm{FeFla}(\mathbf{2})$, c) $\mathrm{Tp}^{\mathrm{Mes}} \mathrm{CoFla}(\mathbf{3})$, d) $\mathrm{Tp}^{\mathrm{Mes}} \mathrm{NiFla} \mathrm{(4)} \mathrm{and} \mathrm{e)} \mathrm{Tp}^{\mathrm{Mes}} \mathrm{ZnFla} \mathrm{(5).}$ 


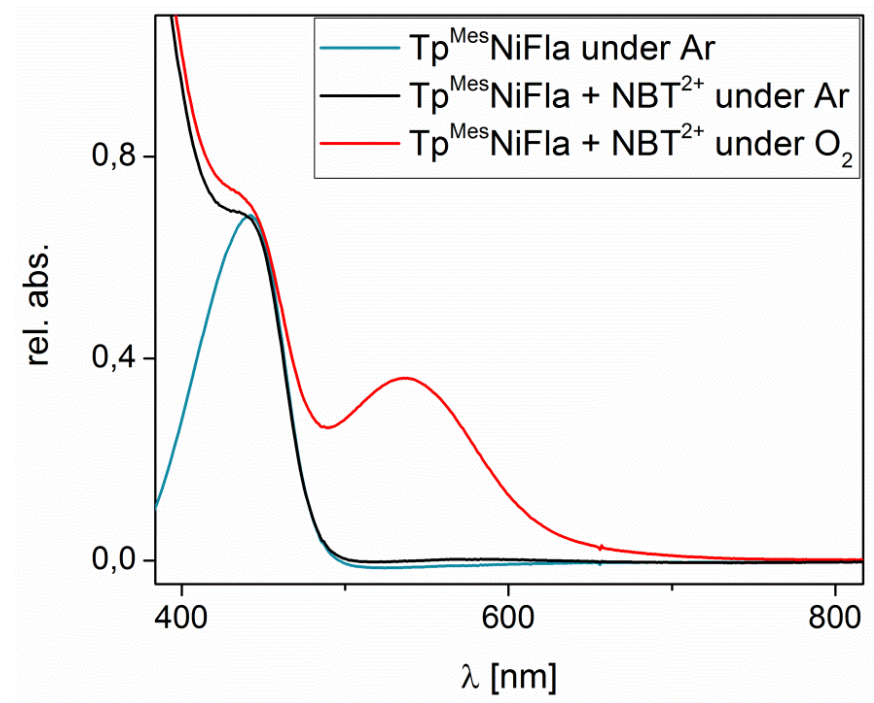

Figure S19: UV-vis spectral change observed for the complex $\mathrm{Tp}^{\mathrm{Mes}} \mathrm{NiFla}(4)\left(0.1 \mathrm{mM}\right.$ in DMF at $80{ }^{\circ} \mathrm{C}$, blue) upon addition of $\mathrm{NBT}^{2+}$ under $\mathrm{Ar}$ (black) and $\mathrm{O}_{2}$ (red).
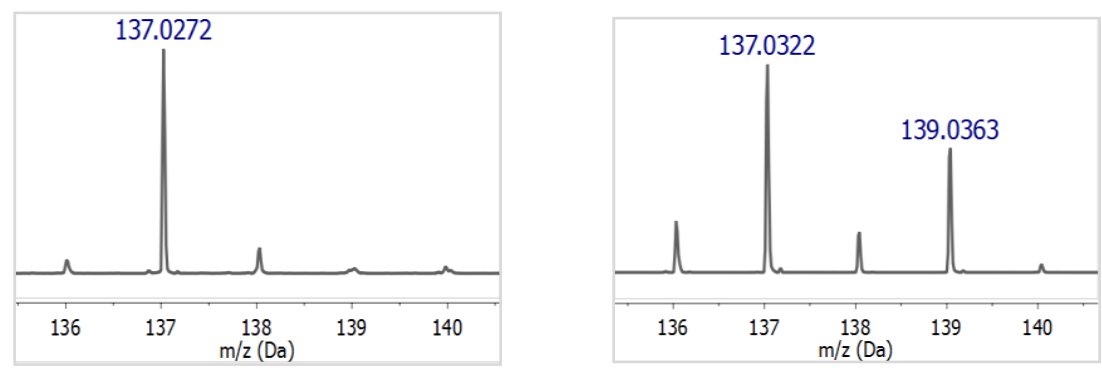

Figure S20: Section of the ESI-MS spectrum after oxygenation reaction with ${ }^{16} \mathrm{O}_{2}$ (left) and ${ }^{18} \mathrm{O}_{2}$ (right) corresponding to salicylic acid with one ${ }^{18} \mathrm{O}$ atom incorporated (calculated values 137.0239 and 139.0281 $m / z$, respectively).

\section{References}

1 Sheldrick, G. M. SADABS; University of Göttingen, Germany, 1996.

2 Sheldrick, G. M. SHELXT - Integrated space-group and crystal-structure determination. Acta Cryst. 2015, A71, 3-8.

3 Sheldrick, G. M. Crystal structure refinement with SHELXL Acta Cryst. 2015, C71, 3-8; Hübschle, C. B.; Sheldrick, G. M.; Dittrich, B. ShelXle: a Qt graphical user interface for SHELXL J. Appl. Cryst. 2011, 44, 1281-1284.

4 Kunrath, F. A.; de Souza, R. F.; Casagrande, O. L.; Brooks, N. R.; Young, V. G. Highly Selective Nickel Ethylene Oligomerization Catalysts Based on Sterically Hindered Tris(pyrazolyl)borate Ligands. Organometallics 2003, 22, 4739-4743. 